Article

\title{
Dosimetric characterization of small radiation therapy electron beams collimated by tubular applicators with the new micro- Silicon detector
}

\author{
S. Russo ${ }^{1, *}$, S. Bettarini ${ }^{2, *}$, B. Grilli Leonulli ${ }^{3}$, M. Esposito ${ }^{1}$, P. Alpi ${ }^{3}$, A. Ghirelli ${ }^{1}$, R. Barca ${ }^{3}$, S. Fondelli ${ }^{3}$, L. \\ Paoletti ${ }^{3}$, S. Pini ${ }^{1}$ and S. Scoccianti ${ }^{3}$
}

1 Medical Physics Unit, Azienda USL Toscana Centro, 50100 Florence, Italy;
2 Specialization School in Medical Physics, University of Florence, Florence, Italy;
3 Radiotherapy Unit, Azienda USL Toscana Centro, 50100 Florence, Italy
* Correspondence: serenella.russo@uslcentro.toscana.it; bettarini.silvia@gmail.com

Featured Application: A complete and accurate dose distribution characterization of small radiation therapy electron beams collimated by tubular applicators is performed by the new generation silicon detector before starting the clinical use.

\begin{abstract}
High-energy small electron beams generated by linear accelerators are used for radiotherapy of localized superficial tumors. The aim of the present study is to assess the dosimetric performance under small radiation therapy electron beams of the novel PTW microSilicon detector by comparison with commercially available dosimeters. Relative dose measurements of circular fields with 20, 30, 40 and $50 \mathrm{~mm}$ aperture diameters were performed for 4 to $12 \mathrm{MeV}$ energy range of electron beams generated by an Elekta Synergy linac. Percentage depth dose, transverse profiles and output factors normalized to the $10 \times 10 \mathrm{~cm}^{2}$ reference field were measured. All dosimetric data were collected in a PTW MP3 motorized water phantom at SSD of $100 \mathrm{~cm}$ by using the novel PTW microSilicon detector. The PTW diode E and the PTW microDiamond were also used in all beam aperture for benchmarking. Data for the biggest field size were also measured by the PTW Advanced Markus ionization chamber. Measurements performed by the microSilicon are in good agreement with the reference values for all the tubular applicators and beam energies, within the stated uncertainties. This confirms the reliability of the microSilicon detector for relative dosimetry of small radiation therapy electron beams collimated by tubular applicators.
\end{abstract}

Keywords: radiotherapy electron beams, output factors, tubular applicator, solid-state detector

\section{Introduction}

Electron beam therapy is a modality for delivering precise radiation doses to localized tumors. The rapid dose fall-off and the short range of an electron beam enable the treatment of target close to the surface, while sparing the underlying tissues. Highenergy electron beams generated by linear accelerators, typically in the range 4 to 20 $\mathrm{MeV}$, are used. Small electron beams suitable for the treatment of limited lesions can be shaped to the target region by using stainless steel circular applicators fastened to the accelerator head with small aperture diameter varying from 20 to $50 \mathrm{~mm}$. A complete and accurate dose distribution characterization of small radiation therapy electron beams collimated by these tubular applicators is mandatory before starting the clinical use. 
However, inherent problems exist in the dosimetry of small and/or irregular electron fields. Small circular collimators affect electron beam side deflections predominated by multiple Coulomb scattering resulting in reduced output. This leads to a lack of lateralscatter equilibrium which plays an important role in small electron beams dosimetry. In fact, when electron fields are smaller than or comparable to the radius required for lateral scattering equilibrium, the depth of maximum adsorbed dose moves toward the surface, the central-axis percentage depth dose decreases, and the output dose/monitor unit decreases [1,2]. Moreover, the size of the detector respect to the field size has to be considered. The detector volume averaging effect, which is dependent on detector size, affects small electron beam dose measurements as well as in narrow photon beams.

Therefore, a reliable tissue-equivalent detector is needed, characterized by a high spatial resolution capability and high sensitivity. Previous studies have largely investigated various parameters such as detector type and volume that affect the dosimetry of photon beams in small fields, leading to the recommendation of the TRS483 on small field dosimetry [3]. However, little attention has been given in literature to small electron beams.

Plane-parallel ionization chambers may be not adequate for small electron beam dosimetry due to their too large collecting electrode and consequent beam nonuniformity over the chamber area. Both silicon diodes and diamond dosimeters were found to be suitable to perform relative electron dosimetry [1,4-5], including the OFs determination. However, only a couple of study reported dosimetric data for beams shaped by tubular applicators [6,7].

The novel PTW microSilicon detector may be an appropriate detector for measurements in high-energy small-field electron beams. The suitability of this small-volume detector for dosimetry in small photon beams was already investigated, showing very good dosimetric properties [8-10]. In addition, three-dimensional characterization of the active volumes of the PTW microSilicon has been performed for accurate Monte Carlo simulations of the detector [11]. Recently, this detector was characterized for electron beam dosimetry of the reference $10 \mathrm{~cm}$ x $10 \mathrm{~cm}$ field [12]. However, to our knowledge, the dosimetric performances of the novel PTW microSilicon detector under small electron beams have not been assessed.

The aim of this work is the dosimetric characterization of clinical electron beams from 4 to $12 \mathrm{MeV}$, generated by a linear accelerator and shaped by commercial tubular applicators, with the novel PTW microSilicon detector by comparison with commercially available dosimeters.

\section{Materials and Methods}

The percentage depth-dose (PDD) curves, transverse beam profiles and output factor (OF) measurements of small-size circular fields were performed for 4 to $12 \mathrm{MeV}$ energy range of electron beams generated by an Elekta Synergy linear accelerator. Electron beams were shaped by stainless steel circular applicators which consist of a main tubular part fastened to the accelerator head and a set of add-on field defining tubes with 20, 30, 40 and $50 \mathrm{~mm}$ aperture diameter at distance from the source of $95 \mathrm{~cm}$.

All dosimetric data were collected during the same measurement session in a PTW MP3 motorized water phantom at SSD of $100 \mathrm{~cm}$, using three different solid-state detectors: a novel PTW microSilicon detector (60023), a PTW diode E (60017) and a PTW microDiamond (60019). Each detector was used in parallel configuration. 
The plane-parallel chamber PTW Advanced Markus was used as reference dosimeter in the $50 \mathrm{~mm}$ diameter circular field and depth dose curves measured by diode $\mathrm{E}$, microDiamond and microSilicon detectors were compared to dose distributions measured by the ion chamber to assess their suitability [12].

\subsection{Detectors}

The PTW microSilicon (60023) is a new unshielded p-type silicon diode detector, well suited for measurements in small electron and photon beams due to its very small sizes (radius $=0.75 \mathrm{~mm}$, thickness $=0.18 \mu \mathrm{m}$ ). The disk-shaped sensitive volume is $0.03 \mathrm{~mm}^{3}$ and the reference point position is on the chamber axis, $0.9 \mathrm{~mm}$ from its tip, which corresponds also to the effective point of measurement for photons. The effective point of measurement for electron beams is located at $0.3 \mathrm{~mm}$ from the chamber tip. No bias voltage applied according to the manufacturer's recommendations.

The PTW microDiamond (60019) is a synthetic single crystal diamond Schottky diode with a disk-shaped sensitive volume of $2.2 \mathrm{~mm}$ in diameter and about $1 \mu \mathrm{m}$ thick. The active volume is located at a water equivalent depth of $1 \mathrm{~mm}$ below the detector surface. The device operates in photovoltaic regime, with no external bias voltage applied. A 5 Gy pre-irradiation was performed.

The PTW diode E (60017) is a previous generation silicon diode detector with a $1 \mathrm{~mm}^{2}$ circular, $30 \mu \mathrm{m}$ thick sensitive volume located on the detector axis, $0.8 \mathrm{~mm}$ below the detector front surface. The water-equivalent window thickness is $1.33 \mathrm{~mm}$. No bias voltage was applied.

The depth ionization distributions measured by the PTW Advanced Markus were converted to depth dose distributions by multiplying the ionization charge at each measurement depth by the stopping-power ratio swair at that depth, according to the IAEA TRS-398 protocol [14]. The variation of ion recombination and polarity effects with depth was also considered.

\subsection{Percentage depth-dose curves and beam profiles}

PDD curves were measured along the beam central axis for the 4, 6, 9 and $12 \mathrm{MeV}$ beam energies and for all field sizes with the three solid-state detectors and with the planeparallel ionization chamber only for the $50 \mathrm{~mm}$ diameter field.

The alignment of each detector with respect to the central axis was initially performed with the PTW Trufix positioning system and then carefully verified by performing both in-plane (i.e. in the gun-target direction) and cross-plane (i.e. perpendicular to the guntarget direction) profile measurements for all the investigated field sizes and for each beam energy.

A shift to the effective point of measurement was used for PDD measurements performed by all the detectors. The scan direction was toward the surface to reduce the effect of meniscus formation and a step size of $0.4 \mathrm{~mm}$ was used for the entire range.

No correction was applied to the PDD curves neither for the diamond nor for the silicon detectors, since the water-to-carbon and water-to-silicon mass collision stopping power ratios are nearly constant in the range 1-20 MeV [14]. 
Transverse beam profiles (in-plane and cross-plane) measurements were performed for each energy and beam aperture with all the three solid-state dosimeters at zref as derived from PDD measurements for the normalization field $10 \mathrm{~cm} \times 10 \mathrm{~cm}$.

PDDs and transverse profiles were acquired and analyzed by the PTW TANDEM electrometer and the PTW Mephysto $\mathrm{MC}^{2}$ software, respectively. No reference detector was used for all dosimetric curve measurements and a step size of $0.4 \mathrm{~mm}$ was used for the entire range. No manipulation was performed before the analysis procedure, except a normalization of all profiles to the central axis.

\subsection{Output factors}

OFs measurements were evaluated for each tubular applicator and for the 4, 6, 9 and 12 $\mathrm{MeV}$ beam energies by the three solid state detectors. The $10 \times 10 \mathrm{~cm}^{2}$ field size was used for the normalization of OFs. A PTW plane-parallel ionization chamber Advanced Markus was also used only for the tubular applicator with $50 \mathrm{~mm}$ aperture diameter.

Each detector was initially centered with the PTW Trufix positioning system in the 3D water scanner. By adopting the methodology recommended in TRS483 [3] for small photon beam dosimetry, two orthogonal profiles were subsequently acquired at the reference depth for each beam energy with the smallest field size by using the three solid state detectors. Then, each detector was positioned at the maximum detector signal point. A step size of $0.2 \mathrm{~mm}$ was used for the entire range to maximize the positioning accuracy.

Output factors were determined as the absorbed dose at zmax for the field of interest $(20,30,40$ and $50 \mathrm{~mm}$ aperture diameter of the tubular applicators) relative to the absorbed dose at zref for the $10 \times 10 \mathrm{~cm}^{2}$ normalization field (reference conditions), at SSD of $100 \mathrm{~cm}$ and with the same number of monitor units as recommended by the TRS398 [14]. For detectors such as diodes and diamonds, the output factor was obtained as the ratio of the detector reading under the non-reference conditions relative to that under reference conditions. When the ionization chamber is used, the variation of water-to-air stopping-power ratio with depth was accounted for in the OF determination. A PTW Unidos E Universal Dosimeter was used for the measurements.

\subsection{Data analysis}

The main parameters obtained from the PDD curves were R100, R80 and R50, i.e. the depths in water of the corresponding maximum value of absorbed dose, of the $80 \%$ and of the half of its maximum value respectively. The practical range $\mathrm{Rp}$ (only for the 50 $\mathrm{mm}$ diameter beam size) and the mean energy at the phantom surface E0 were also determined.

The difference in \% over the entire depth range and the difference in distance $(\mathrm{mm})$ within the region of the curve maximum slope (20\% - $80 \%)$ were evaluated to compare PDDs for all beam energies: for the $50 \mathrm{~mm}$ diameter tubular applicator the differences between each solid-state dosimeter and the ionization chamber were evaluated, for the remaining tubular collimators the diode $\mathrm{E}$ was chosen as the reference detector.

The profiles measured by the different solid-state detectors were compared in terms of field size evaluated by the full-width half maximum (FWHM) and in terms of the $80 \%-$ $20 \%$ penumbra obtained by averaging the left- and right-hand side values. 
Similarly to the PDD case, the difference in \% over the entire off axis range and the difference in distance $(\mathrm{mm})$ within the penumbra region were evaluated between the profiles measured by the diode $\mathrm{E}$ and the other two solid state detectors, the microDiamond and the microSilicon, respectively.

For OFs evaluation, the reference detector choice was dissimilar according to the tubular applicator size: for the $50 \mathrm{~mm}$ aperture diameter the ionization chamber was considered as the reference, while for the other beam diameters $(20,30$ and $40 \mathrm{~mm})$ the diode $\mathrm{E}$ was considered as the reference. Relative differences between OF values, measured by each detector and the reference one, were calculated.

Comparisons of dose parameters measured by the reference detector and other dosimeters were performed using a two-tailed Wilcoxon signed-rank test, considering a p-value of $<0.05$ as significant.

\section{Results}

\subsection{Percentage depth-dose curves and beam profiles measurements}

Figure 1 shows the PDD curves measured for all beam energies with the three solidstate detectors and the plane-parallel ionization chamber for the $50 \mathrm{~mm}$ diameter field. The differences in \% between PDDs measured by the solid-state dosimeters and the ionization chamber are also shown. An agreement of $\pm 3 \%$ was observed among the detector responses for all the beam energies over the whole PDD curve with the exception of the region in close proximity to the water surface, where the detectors are partially in air. The difference grown to about $5 \%$ in the maximum dose gradient area only for the $12 \mathrm{MeV}$ energy beam. The maximum difference in distance $(\mathrm{mm})$ within the $20 \%-80 \%$ region, reported in table 4, for each solid-state detector relative to the reference data, was $0.7 \mathrm{~mm}$ for all beam energies, except for $12 \mathrm{MeV}$ where $1.3 \mathrm{~mm}$ is the maximum distance. As far as concerned only the diode $\mathrm{E}$, the maximum difference in distance was $0.4 \mathrm{~mm}$ in all curves. No statistically significant differences were found between the reference data and those measured by the other detectors.

For the remaining tubular collimators, the maximum difference in \% over the entire depth range was within $4 \%$ and the maximum difference in distance within the region of the curve maximum slope was $1.1 \mathrm{~mm}$ for all beam energies. Similar results were obtained for the microDiamond and microSilicon detectors. The PDD curves measured by the three solid-state detectors in the $20 \mathrm{~mm}$ diameter field for all beam energies are reported in Figure 2, together with the respective percentage differences between PDDs measured by the microDiamond and by the microSilicon dosimeters and the diode $\mathrm{E}$ data. 


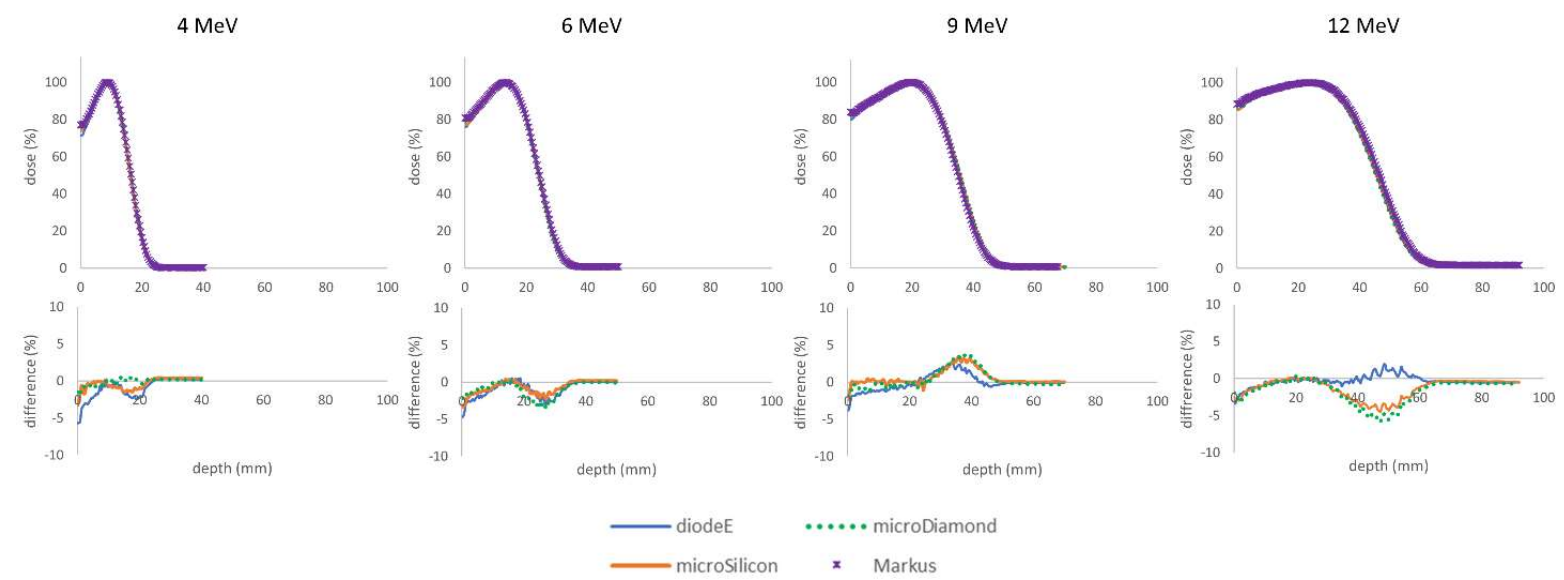

Figure 1. In the upper part: PDD curves measured for all beam energies with the three solid-state detectors and the plane-parallel ionization chamber for the $50 \mathrm{~mm}$ diameter field. In the bottom part: \% differences between PDDs measured by the solid-state dosimeters and the ionization chamber.

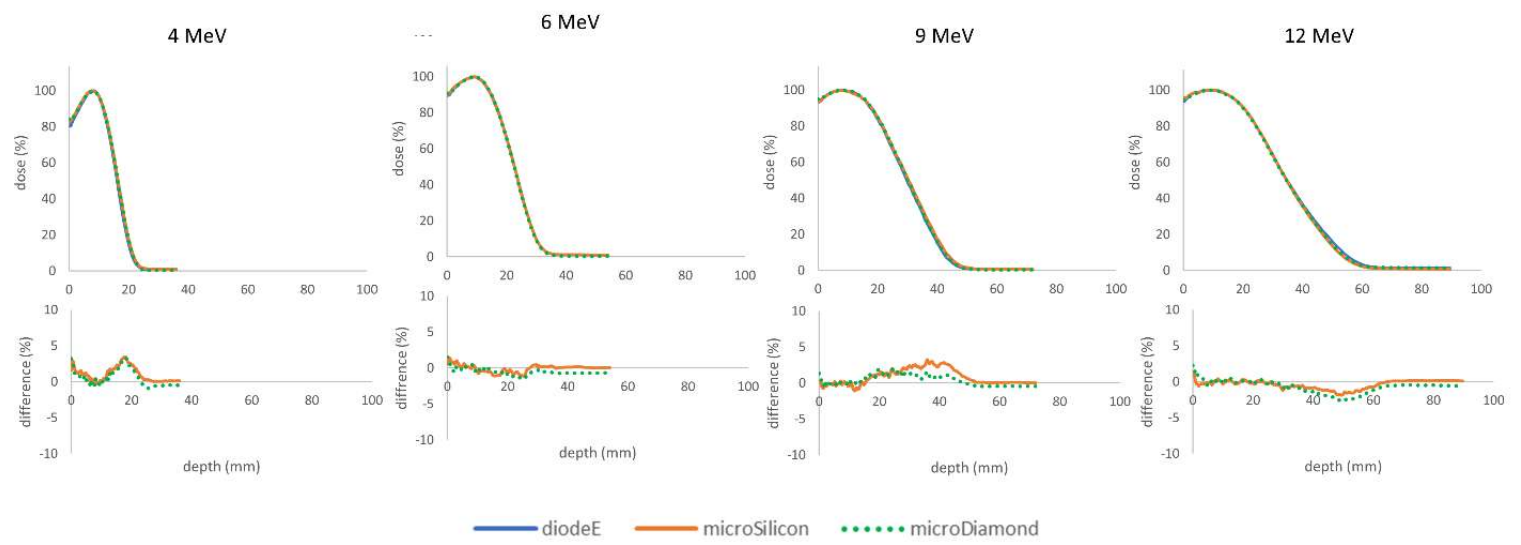

Figure 2: PDD curves measured for the $20 \mathrm{~mm}$ diameter field for all beam energies with the solid-state detectors: \% differences between microSilicon and microDiamond data and diode E reference measurements were also shown.

Table 1. Most relevant dosimetric parameters extracted from PDD curves for the $50 \mathrm{~mm}$ diameter field, measured by ionization chamber Markus (M), diode E (d-E), microDiamond (m-D) and microSilicon (m-Si)

\begin{tabular}{|c|c|c|c|c|c|c|c|c|c|c|c|c|c|c|c|c|c|c|c|c|}
\hline \multirow[b]{2}{*}{$\begin{array}{l}\text { Energy } \\
(\mathrm{MeV})\end{array}$} & \multicolumn{4}{|c|}{$\mathrm{R} 100$ (mm) } & \multicolumn{4}{|c|}{$\mathrm{R} 80$ (mm) } & \multicolumn{4}{|c|}{$\mathrm{R} 50$ (mm) } & \multicolumn{4}{|c|}{$\mathrm{Rp}(\mathrm{mm})$} & \multicolumn{4}{|c|}{ E0 $(\mathrm{MeV})$} \\
\hline & M & $\mathrm{d}-\mathrm{E}$ & $\begin{array}{l}\text { m- } \\
\text { D }\end{array}$ & $\begin{array}{l}\mathrm{m}- \\
\mathrm{Si}\end{array}$ & M & $d-E$ & $\begin{array}{l}\mathrm{m}- \\
\mathrm{D}\end{array}$ & $\begin{array}{l}\mathrm{m}- \\
\mathrm{Si}\end{array}$ & $\mathrm{M}$ & $d-E$ & $\begin{array}{l}\mathrm{m}- \\
\mathrm{D}\end{array}$ & $\begin{array}{l}\mathrm{m}- \\
\mathrm{Si}\end{array}$ & M & $d-E$ & $\begin{array}{l}\mathrm{m}- \\
\mathrm{D}\end{array}$ & $\begin{array}{l}\mathrm{m}- \\
\mathrm{Si}\end{array}$ & $\mathrm{M}$ & $d-E$ & $\begin{array}{l}\mathrm{m}- \\
\mathrm{D}\end{array}$ & $\begin{array}{l}\mathrm{m}- \\
\mathrm{Si}\end{array}$ \\
\hline 4 & 8.4 & 9.2 & 8.8 & 8.8 & 13.4 & 13.4 & 13.3 & 13.5 & 16.5 & 16.3 & 16.3 & 16.5 & 21.1 & 20.8 & 20.9 & 21.2 & 3.8 & 38 & 3.8 & 3.8 \\
\hline 6 & 13.2 & 12.8 & 13.6 & 12.8 & 20.2 & 20.1 & 20.0 & 19.8 & 24.4 & 24.1 & 24.1 & 24.0 & 30.8 & 30.1 & 30.5 & 30.2 & 5.7 & 5.6 & 5.6 & 5.6 \\
\hline 9 & 19.6 & 20.0 & 19.6 & 19.6 & 29.5 & 29.9 & 29.8 & 29.9 & 35.1 & 35.4 & 35.6 & 35.6 & 43.5 & 43.3 & 44.0 & 44.1 & 8.2 & 8.3 & 8.3 & 8.3 \\
\hline 12 & 23.2 & 22.8 & 24.0 & 224 & 38.9 & 38.9 & 38.1 & 37.9 & 46.6 & 46.7 & 45.7 & 45.4 & 57.0 & 58.1 & 56.4 & 56.1 & 10.9 & 10.9 & 10.6 & 10.6 \\
\hline
\end{tabular}


Table 1 summarizes the most relevant dosimetric parameters extracted from PDD curves for the $50 \mathrm{~mm}$ diameter field and all beam energies, showing a maximum deviation of about $1 \mathrm{~mm}$ for all the investigated depth parameters and $0.3 \mathrm{MeV}$ for E0 measured by the solid-state detectors respect to the plane-parallel ionization chamber.

The dosimetric characteristics of all cutouts, except for the maximum diameter field, and all beam energies are reported in Table 2: a maximum deviation of $1.6 \mathrm{~mm}$ on Dmax and of $0.8 \mathrm{~mm}$ for all the other depth parameters investigated and $0.2 \mathrm{MeV}$ for E0 were found between microDiamond and microSilicon measurements and the diode $\mathrm{E}$ data. The electron beam parameters R100, R80, and R50 shifted toward the phantom surface with decreasing field dimensions, in particular for the smallest field size. Furthermore, these parameters increased with increasing energy and penetrate deeper into the phantom.

Table 2: Most relevant dosimetric parameters derived from PDD curves for all tubular applicators, measured by the solid-state dosimeters diode $\mathrm{E}(\mathrm{d}-\mathrm{E})$, microDiamond $(\mathrm{m}-\mathrm{D})$ and microSilicon $(\mathrm{m}-\mathrm{Si})$

Diameter $20 \mathrm{~mm}$

\begin{tabular}{|c|c|c|c|c|c|c|c|c|c|c|c|c|}
\hline \multirow[b]{2}{*}{$\begin{array}{l}\text { Energy } \\
(\mathrm{MeV})\end{array}$} & \multicolumn{3}{|c|}{ R100 (mm) } & \multicolumn{3}{|c|}{$\mathrm{R} 80$ (mm) } & \multicolumn{3}{|c|}{ R 50 (mm) } & \multicolumn{3}{|c|}{$\mathrm{E} 0(\mathrm{MeV})$} \\
\hline & $\mathrm{d}-\mathrm{E}$ & $\mathrm{m}-\mathrm{D}$ & $\mathrm{m}-\mathrm{Si}$ & $\mathrm{d}-\mathrm{E}$ & $\mathrm{m}-\mathrm{D}$ & $\mathrm{m}-\mathrm{Si}$ & $d-E$ & $\mathrm{~m}-\mathrm{D}$ & $\mathrm{m}-\mathrm{Si}$ & $\mathrm{d}-\mathrm{E}$ & $\mathrm{m}-\mathrm{D}$ & $\mathrm{m}-\mathrm{Si}$ \\
\hline 4 & 8.4 & 8.0 & 8.0 & 13.0 & 13.2 & 13.1 & 16.2 & 16.5 & 16.4 & 4.4 & 4.5 & 4.5 \\
\hline 6 & 9.6 & 9.2 & 8.8 & 17.4 & 17.2 & 17.3 & 22.7 & 22.6 & 22.5 & 5.3 & 5.3 & 5.2 \\
\hline 9 & 7.6 & 7.2 & 7.6 & 21.3 & 21.6 & 21.7 & 29.7 & 30.4 & 30.0 & 6.9 & 7.1 & 7.0 \\
\hline 12 & 10.0 & 8.5 & 8.8 & 24.5 & 24.5 & 24.4 & 34.9 & 34.8 & 34.6 & 8.1 & 8.1 & 8.1 \\
\hline \multicolumn{13}{|c|}{ Diameter $30 \mathrm{~mm}$} \\
\hline & \multicolumn{3}{|c|}{$\mathrm{R} 100$ (mm) } & \multicolumn{3}{|c|}{$\mathrm{R} 80$ (mm) } & \multicolumn{3}{|c|}{ R 50 (mm) } & \multicolumn{3}{|c|}{ E0 $(\mathrm{MeV})$} \\
\hline $\begin{array}{c}\text { Energy } \\
(\mathrm{MeV})\end{array}$ & $d-E$ & $\mathrm{~m}-\mathrm{D}$ & $\mathrm{m}-\mathrm{Si}$ & $d-E$ & $\mathrm{~m}-\mathrm{D}$ & $\mathrm{m}-\mathrm{Si}$ & $d-E$ & $\mathrm{~m}-\mathrm{D}$ & $\mathrm{m}-\mathrm{Si}$ & $\mathrm{d}-\mathrm{E}$ & $\mathrm{m}-\mathrm{D}$ & $\mathrm{m}-\mathrm{Si}$ \\
\hline 4 & 8.6 & 8.6 & 8.8 & 13.3 & 13.3 & 13.3 & 16.3 & 16.4 & 16.4 & 4.4 & 4.4 & 4.4 \\
\hline 6 & 12.0 & 12.0 & 11.6 & 19.5 & 19.5 & 19.3 & 24.1 & 23.9 & 23.7 & 5.6 & 5.6 & 5.5 \\
\hline 9 & 14.4 & 13.6 & 14.0 & 27.0 & 26.6 & 26.5 & 34.1 & 34.0 & 33.8 & 7.9 & 7.9 & 7.9 \\
\hline 12 & 16.8 & 15.6 & 15.6 & 32.0 & 32.1 & 31.9 & 42.3 & 42.1 & 41.7 & 9.9 & 9.8 & 9.7 \\
\hline \multicolumn{13}{|c|}{ Diameter $40 \mathrm{~mm}$} \\
\hline & \multicolumn{3}{|c|}{$\mathrm{R} 100$ (mm) } & \multicolumn{3}{|c|}{$\mathrm{R} 80$ (mm) } & \multicolumn{3}{|c|}{$\mathrm{R} 50$ (mm) } & \multicolumn{3}{|c|}{ E0 $(\mathrm{MeV})$} \\
\hline $\begin{array}{l}\text { Energy } \\
(\mathrm{MeV})\end{array}$ & $d-E$ & $\mathrm{~m}-\mathrm{D}$ & $\mathrm{m}-\mathrm{Si}$ & $d-E$ & m-D & $\mathrm{m}-\mathrm{Si}$ & $d-E$ & $\mathrm{~m}-\mathrm{D}$ & $\mathrm{m}-\mathrm{Si}$ & $\mathrm{d}-\mathrm{E}$ & $\mathrm{m}-\mathrm{D}$ & $\mathrm{m}-\mathrm{Si}$ \\
\hline 4 & 9.2 & 8.8 & 9.2 & 13.6 & 13.9 & 13.7 & 16.5 & 16.8 & 16.7 & 3.8 & 3.9 & 3.9 \\
\hline 6 & 13.6 & 13.6 & 12.0 & 20.1 & 20.0 & 19.8 & 24.2 & 24.2 & 23.9 & 5.6 & 5.6 & 5.6 \\
\hline 9 & 19.6 & 18.8 & 20.0 & 29.5 & 29.5 & 29.5 & 35.4 & 35.8 & 35.6 & 8.2 & 8.3 & 8.3 \\
\hline 12 & 19.5 & 18.0 & 19.4 & 36.3 & 36.2 & 36.0 & 45.1 & 44.7 & 44.3 & 10.5 & 10.4 & 10.3 \\
\hline
\end{tabular}

The crossline profiles measured by the different solid-state detectors and the difference in $\%$ over the entire off axis range between microDiamond and microSilicon measurements and the diode E data for the smallest circular applicator were shown in Figure 3. Both for in-plane and cross-plane profiles, for all tubular applicators and beam energies, the maximum difference in $\%$ over the entire range was within $1 \%$ in the low gradient regions and grew up to $3 \%$ in the maximum slope area. Furthermore, in these regions, the maximum 
difference in distance was within $0.4 \mathrm{~mm}$. No statistically significant differences were found between the reference data and microDiamond or microSilicon results.

In Table 3 the field size, evaluated by the FWHM, and the $80 \%-20 \%$ penumbra values for all applicators and beam energies were reported: a maximum deviation of $0.4 \mathrm{~mm}$ in field size and penumbra evaluation was found for both microDiamond and microSilicon respect to the diode E, without significative difference between the two detectors.

\subsection{OF measurements}

The OF values obtained using all the dosimeters for all the tubular applicators and beam energies are shown in Figure 4. OF values were reported as a function of nominal field size, defined as the applicator diameter. OFs measured by the solid-state detectors agreed with those measured with the ionization chamber within $1.7 \%$ for the $50 \mathrm{~mm}$ diameter, as can be seen in table 5. Noteworthy that the microSilicon got the best agreement with the ionization chamber, with a maximum deviation of $0.8 \%$.

For the 20, 30 and $40 \mathrm{~mm}$ applicator diameters, OFs agreement between the microSilicon data and the reference values was always within $1.2 \%$. All OF values measured by microDiamond well agreed with the reference data: differences were typically within $1.4 \%$ with a maximum value of $2.5 \%$ for $4 \mathrm{MeV}$ and the smallest aperture.
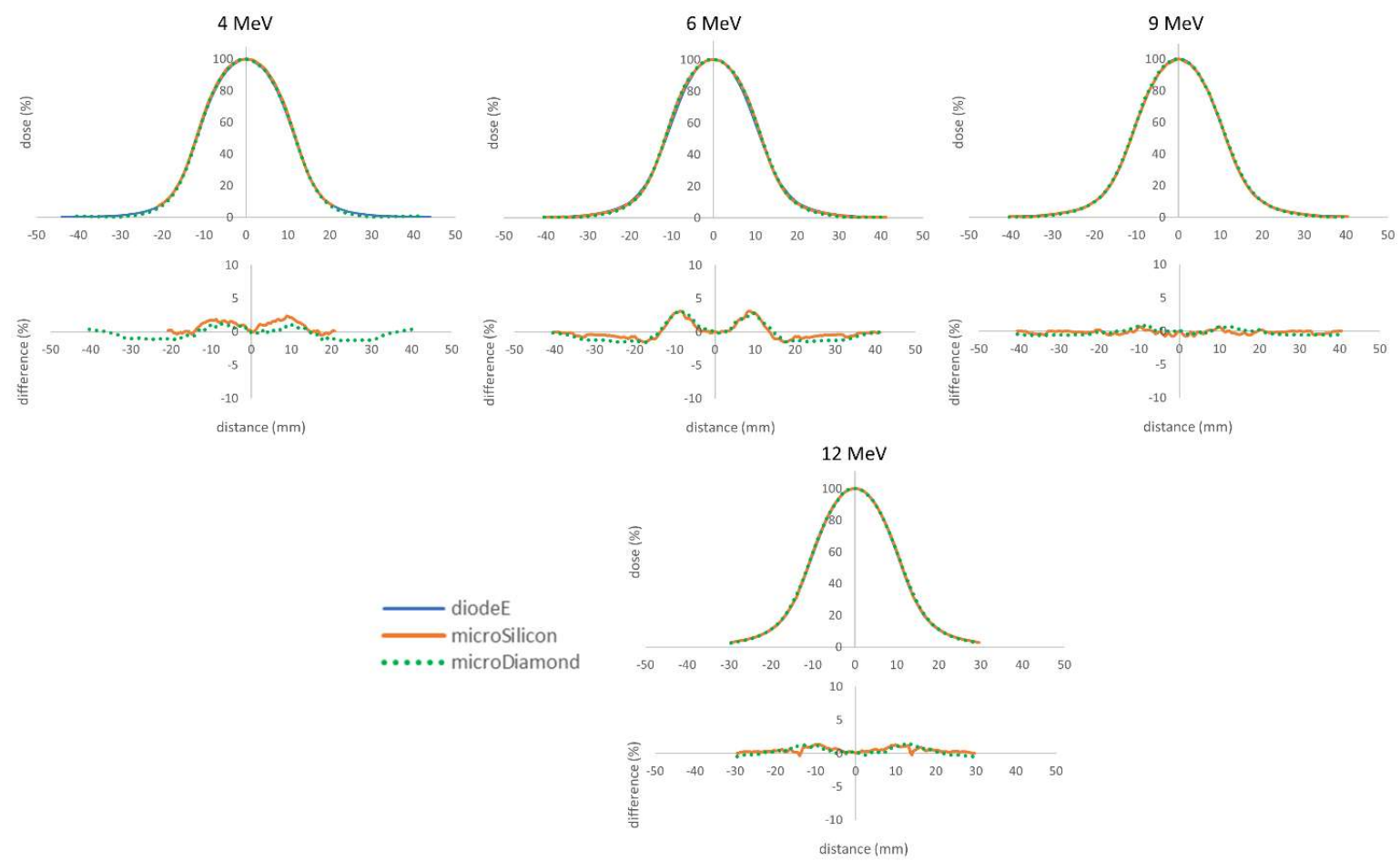

Figure 3: Crossline beam profiles measured by the different solid-state detectors and \% respective difference over the entire off axis range between microDiamond and microSilicon measurements and diode $\mathrm{E}$ data. 
Table 3: FWHM and $80 \%-20 \%$ penumbra values for all circular applicators, measured by the solid-state dosimeters diode $\mathrm{E}(\mathrm{d}-\mathrm{E})$, microDiamond (m-D) and microSilicon (m-Si).

\begin{tabular}{|c|c|c|c|c|c|c|c|c|c|c|c|c|}
\hline \multirow{3}{*}{$\begin{array}{c}\text { field } \\
\text { diameter } \\
(\mathrm{mm})\end{array}$} & \multicolumn{12}{|c|}{ Field size (mm) } \\
\hline & \multicolumn{3}{|c|}{$4 \mathrm{MeV}$} & \multicolumn{3}{|c|}{$6 \mathrm{MeV}$} & \multicolumn{3}{|c|}{$9 \mathrm{MeV}$} & \multicolumn{3}{|c|}{$12 \mathrm{MeV}$} \\
\hline & $d-E$ & $\mathrm{~m}-\mathrm{D}$ & $\mathrm{m}-\mathrm{Si}$ & $\mathrm{d}-\mathrm{E}$ & $m-D$ & $\mathrm{~m}-\mathrm{Si}$ & $d-E$ & $\mathrm{~m}-\mathrm{D}$ & $\mathrm{m}-\mathrm{Si}$ & $\mathrm{d}-\mathrm{E}$ & $\mathrm{m}-\mathrm{D}$ & $\mathrm{m}-\mathrm{Si}$ \\
\hline 50 & 54.3 & 54.3 & 54.5 & 53.5 & 53.6 & 53.7 & 52.8 & 52.8 & 53.0 & 52.9 & 52.9 & 53.0 \\
\hline 40 & 43.4 & 43.7 & 43.6 & 42.7 & 43.0 & 43.1 & 42.3 & 42.5 & 42.4 & 42.2 & 42.5 & 42.6 \\
\hline 30 & 32.9 & 33.1 & 33.2 & 32.9 & 32.5 & 32.5 & 31.8 & 32.0 & 32.0 & 31.7 & 32.0 & 32.0 \\
\hline \multirow[t]{2}{*}{20} & 23.4 & 23.6 & 23.8 & 22.9 & 23.2 & 23.1 & 22.7 & 22.8 & 22.6 & 22.4 & 22.7 & 22.7 \\
\hline & \multicolumn{12}{|c|}{ Penumbra (mm) } \\
\hline field & & $4 \mathrm{MeV}$ & & & $6 \mathrm{MeV}$ & & & $9 \mathrm{MeV}$ & & & $12 \mathrm{Me}$ & \\
\hline $\begin{array}{c}\text { diameter } \\
(\mathrm{mm})\end{array}$ & $\mathrm{d}-\mathrm{E}$ & $\mathrm{m}-\mathrm{D}$ & $\mathrm{m}-\mathrm{Si}$ & $\mathrm{d}-\mathrm{E}$ & $\mathrm{m}-\mathrm{D}$ & m-Si & $d-E$ & $\mathrm{~m}-\mathrm{D}$ & $\mathrm{m}-\mathrm{Si}$ & $d-E$ & $\mathrm{~m}-\mathrm{D}$ & $\mathrm{m}-\mathrm{Si}$ \\
\hline 50 & 11.5 & 11.4 & 11.4 & 11.6 & 11.8 & 11.8 & 12.3 & 12.4 & 12.0 & 12.0 & 12.5 & 12.3 \\
\hline 40 & 10.9 & 10.9 & 10.8 & 11.0 & 11.0 & 10.8 & 11.5 & 11.6 & 11.3 & 11.6 & 11.9 & 11.6 \\
\hline 30 & 10.4 & 10.1 & 10.1 & 10.4 & 10.2 & 10.2 & 10.9 & 10.7 & 10.7 & 10.9 & 11.1 & 11.1 \\
\hline 20 & 8.9 & 8.6 & 8.6 & 9.0 & 8.8 & 8.8 & 9.5 & 9.4 & 9.4 & 9.6 & 9.8 & 9.7 \\
\hline
\end{tabular}

Table 4: Maximum difference in distance $(\mathrm{mm})$ within the 20\%- 80\% PDD region for the solid-state detectors (diode $\mathrm{E}$ reported as $\mathrm{d}-\mathrm{E}$, microDiamond as $\mathrm{m}-\mathrm{D}$ and microSilicon as $\mathrm{m}$-Si) respect to the chosen reference for each beam energy. The mean value over all beam energies was also shown.

difference in distance $(\mathrm{mm})$

\begin{tabular}{cccccccccc}
\hline & \multicolumn{2}{c}{$20 \mathrm{~mm}$} & \multicolumn{2}{c}{$30 \mathrm{~mm}$} & \multicolumn{2}{c}{$40 \mathrm{~mm}$} & \multicolumn{3}{c}{$50 \mathrm{~mm}$} \\
Energy (MeV) & $\mathrm{m}-\mathrm{D}$ & $\mathrm{m}-\mathrm{Si}$ & $\mathrm{m}-\mathrm{D}$ & $\mathrm{m}-\mathrm{Si}$ & $\mathrm{m}-\mathrm{D}$ & $\mathrm{m}-\mathrm{Si}$ & $\mathrm{d}-\mathrm{E}$ & $\mathrm{m}-\mathrm{D}$ & $\mathrm{m}-\mathrm{Si}$ \\
\hline 4 & 0.3 & 0.4 & 0.2 & 0.1 & 0.3 & 0.4 & 0.3 & 0.03 & 0.1 \\
6 & 0.2 & 0.1 & 0.3 & 0.1 & 0.4 & 0.1 & 0.4 & 0.4 & 0.3 \\
9 & 0.4 & 0.9 & 0.4 & 0.4 & 0.5 & 0.7 & 0.4 & 0.7 & 0.6 \\
12 & 0.5 & 0.8 & 1.1 & 0.4 & 1.1 & 0.6 & 0.3 & 1.3 & 0.9 \\
mean & 0.4 & 0.6 & 0.5 & 0.3 & 0.6 & 0.5 & 0.4 & 0.6 & 0.5
\end{tabular}




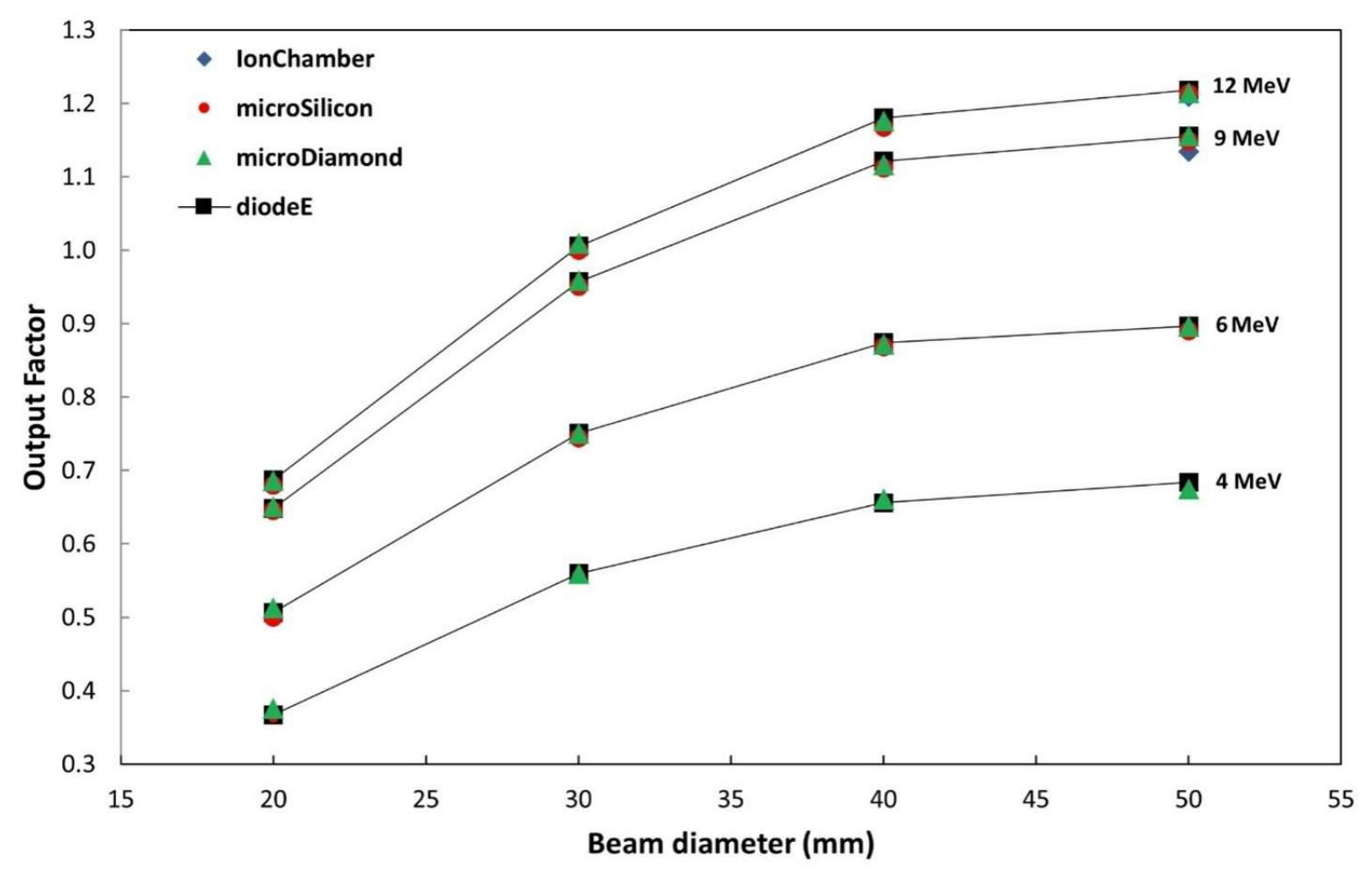

Figure 4: Output factor values measured by all dosimeters for all aperture diameters and beam energies. Measurement uncertainties were within the sizes of point indicators

Table 5: OF values measured for all beam energies with all the dosimeters for the $50 \mathrm{~mm}$ diameter tubular applicator (ionization chamber Markus reported as $\mathrm{M}$, diode $\mathrm{E}$ as $\mathrm{D}-\mathrm{E}$, microDiamond as $\mathrm{m}-\mathrm{D}$ and microSilicon as $\mathrm{m}-\mathrm{Si}$ ) and only with solid-state dosimeters for the remaining applicators.

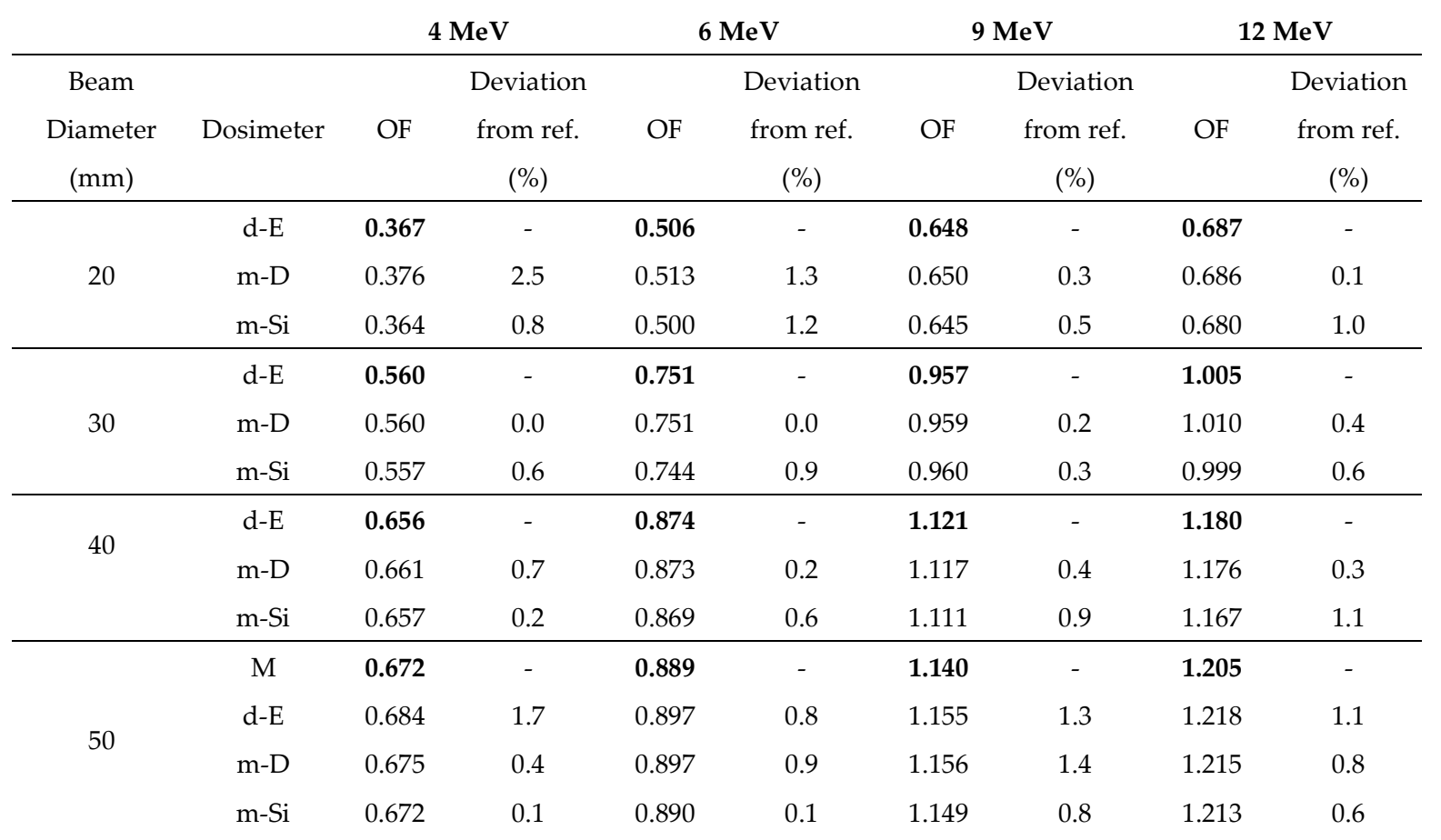




\subsection{Uncertainty budget evaluation}

The maximum uncertainty related to detector positioning over the whole PDD curve was estimated to be in the range $2-4 \%$ for all the beam energies and field sizes. This finding was related to the used scanning step size of $0.4 \mathrm{~mm}$ and to the evaluated dose gradient of about $4 \% \mathrm{~mm}^{-1}$ for the higher beam energy and $9 \% \mathrm{~mm}^{-1}$ for the lower beam energy, regardless of the field size.

For all the profile curves, the maximum uncertainty related to detector positioning was estimated to be within $0.4 \%$ in the center area and 3\% in the maximum slope area, for all beam energies and field sizes, given the step size of $0.4 \mathrm{~mm}$. Moreover, another component of the uncertainties of about $1 \%$ must be introduced to take into account that the reference detector was not used for relative dosimetry.

A complete total uncertainty budget was estimated for OF determination considering four main components: the position uncertainty of the detector, the uncertainty related to the electrometer, the statistical dispersion of repeated measurements and the reproducibility of the OF values, obtained in different measurement sessions.

The positioning accuracy was evaluated to be $\pm 0.2 \mathrm{~mm}$, considering the method used to identify the center field, and the uncertainty related to detector positioning was estimated to be lower than $0.1 \%$. The accuracy of the electrometer used, supplied by the manufacturer, was $\pm 0.2 \%$. The measurement readout uncertainties (calculated as one standard deviation of the mean of repeated measurements) was lower than $0.5 \%$ for solid-state detectors and $0.2 \%$ for the ionization chamber. The reproducibility of the OF values, obtained in different measurement sessions, was about 1\% (1 SD) for the solid-state dosimeters and about $0.5 \%$ ( $1 \mathrm{SD}$ ) for the ionization chamber.

Using Gaussian error propagation, the combined uncertainties for the measurements were estimated to be about $1.5 \%$ for the solid-state dosimeters and $1 \%$ for the ionization chamber.

\section{Discussion}

Radiation therapy electron beams collimated by tubular applicators are widely used for the treatment of small superficial lesions because of their uniform dose distributions at the surface shaped to the target region and their rapid decrease in the dose less than a specific depth. For small circular electron beams, measurements of outputs, depth dose curves and lateral beam profiles are necessary for a complete dosimetric characterization of each tubular applicator. However, the electron disequilibrium and high-gradient dosing make the small electron beam dosimetry challenging. Diode detectors are considered suitable for measurements of the scanning data and the relative output factors in small electron beams [13].

The microSilicon detector has been recently introduced to overcome the performance of its predecessor diode $\mathrm{E}$ with an optimization of the basic dosimetric properties such as higher dose stability, lower dose per pulse dependence and smaller sensitivity to temperature. For this purpose, the entrance window was rendered more water equivalent by reducing the density of the casting compound on top of the silicon chip compared to the predecessor [11]. 
The lack of investigation of the microSilicon detector performance in high-energy small electron beams boosted the need for the characterization of clinical electron beams shaped by commercial tubular applicators performed in this study.

According to IAEA 398, plane-parallel air-filled ionization chambers are recommended to be used for PDD measurements at all electron energies and, below $10 \mathrm{MeV}$, their use is mandatory. However, in small beam dosimetry the diameter of the collecting electrode should be considered in order to prevent the influence of radial non-uniformities of the beam profile [14]. TG25 suggested that measurements can be done with an ionization chamber that is small enough such that its active volume fits in the flat portion of the beam [1,13]. Diode and diamond detectors can be used as an alternative to the ionization chamber after verifying that the detector is suitable for depth dose measurements trough test comparisons at a representative beam quality [14].

To this purpose, the Advanced Markus ionization chamber has been used as reference for PDD and OF measurements for the $50 \mathrm{~mm}$ diameter applicator and the differences between each solid-state dosimeter and the ionization chamber were evaluated. Diode E detector presented the overall minimum difference in dose and distance with the ion chamber data and silicon diode detectors have been considered suitable for electron dosimetry for a long time in the literature $[1,13]$. So far, the diode $\mathrm{E}$ was chosen as the reference detector for the remaining tubular collimators to compare measurements performed by the microSilicon and the microDiamond.

The dose difference in \% over the entire depth range was evaluated for PDD. However, the dose difference may not be appropriate to compare the variability among detectors in the region between the R80 and the R20, where the dose gradient is higher. So, the distance-to-agreement representing the minimum distance to the same value from the reference data was calculated for the solid-state detectors.

We found that differences between solid-state detectors are still consistent with the measurement uncertainty, taking into account the step size used for data acquisition and the absence of the reference detectors due to the small size of the investigated fields.

The results reported in table 4, regarding the maximum distance-to-agreement averaged over all beam energies between the ion chamber and each solid-state dosimeter data, were found to be similar to the ones presented by Akino et al. [12]. In particular, for the $50 \mathrm{~mm}$ tubular applicator our results were $0.4 \mathrm{~mm}, 0.6 \mathrm{~mm}$ and $0.5 \mathrm{~mm}$ for diode $\mathrm{E}$, microDiamond and microSilicon respectively. Similarly, for the same solid-state detectors, Akino et al. [12] obtained $0.51 \mathrm{~mm}, 0.68 \mathrm{~mm}$ and $0.86 \mathrm{~mm}$ respectively in the $10 \times 10 \mathrm{~cm} 2$ reference field size.

In this study transverse beam profiles measurements were performed for each energy and beam aperture at zref, as derived for the reference field $10 \times 10 \mathrm{~cm} 2$, with all the three solid-state dosimeters. We decided to measure all beam profiles at the same depth for a better comparison of profile parameters since field size and penumbra values are very sensitive to the measurement depth. A maximum deviation of $0.4 \mathrm{~mm}$ in penumbra evaluation was found in our measurements. Differently, Bagalà et al. acquired beam profiles at the depth of maximum dose, as derived from PDD measurements for different detectors, and found a maximum difference up to $1.1 \mathrm{~mm}$ in penumbra values for beam profiles measured at slightly different depths [6].

A decrease of the OF value is observed for each field size by decreasing the beam energy and for each energy by reducing the field size. Accordingly, the OF for the smallest field size and the lower energy was about 3 times lower than the reference field size. OFs 
larger than unity were observed at $9 \mathrm{MeV}$ and $12 \mathrm{MeV}$, with an increase for the $50 \mathrm{~mm}$ diameter field size at $12 \mathrm{MeV}$ up to $20 \%$ in comparison with the output of the $10 \times 10$ $\mathrm{cm} 2$ field size. The same behaviour was observed by Di Venanzio et al. [5] with an increase for the $50 \mathrm{~mm}$ diameter field size at $15 \mathrm{MeV}$ up to $30 \%$.

OF determined in this study shown a moderate to high difference with the values measured by Venanzio et al. [5]: for all field sizes at $6 \mathrm{MeV}$ beam energy deviations were within $3 \%$, but an increase up to about 30\% was observed for $12 \mathrm{MeV}$ beam energy and the $20 \mathrm{~mm}$ beam diameter. This confirms what reported in literature that is that different components determine the linac output when tubular applicators are used. Therefore, dosimetric measurements should be performed for each condition foreseen for patient irradiation, using reliable high-resolution detectors [5].

\section{Conclusions}

Beam output in small electron beams shaped by tubular applicators is influenced by electron scattering from applicator walls, air enclosed by applicator and scattering in the water. Dosimetric parameters in such fields, in particular OFs, differ a lot from those of fields shaped by typical electron applicators. Noteworthy, it is recommended to measure dosimetric parameters for each field separately rather than attempt to interpolate values. The selection of a suitable dosimeter for the measurement of electron parameters in small fields with lateral scatter disequilibrium is a critical issue. Moreover, the methodology used for accurate measurements of dose curves and beam output is also a fundamental aspect in the dosimetry of small electron beams.

In this study dose profiles, PDDs and OFs measured by the PTW microSilicon detector were found to be in good agreement with reference detector measurements for all the tubular applicators and beam energies, within the stated uncertainties. This confirm that PTW microSilicon is a suitable detector for the dosimetry of small electron beams collimated with circular applicators and can be considered as a reliable alternative to its predecessor diode E.

Author Contributions: Conceptualization, SR and SB; methodology, SR; software, SB; validation, BGL, RB, LP, SS, PA, SF; formal analysis, SB; investigation, SB; data curation, SR, SB; writing - original draft preparation, SR, SB; writing - review and editing, ME, SP, AG; supervision, SS;

Funding: This research received no external funding.

Conflicts of Interest: The authors declare no conflict of interest.

\section{References}

1. Gerbi, B.J.; Antolak, J.A.; Deibel, F.C.; Followill, D.S.; Herman, M.G.; Higgins, P.D., Huq, M.S.; Mihailidis, D.N.; Yorke, E.D.; Hogstrom, K.R.; Khan, F.M. Recommendations for clinical electron beam dosimetry: Supplement to the recommendations of Task Group 25. Medical Physics 2009, 36, 3239-3279; DOI:10.1118/1.3125820.

2. Xu, M.; Sethi, A.; Glasgow, G.P.; Dosimetry of small circular fields for 6-MeV electron beams. Medical Dosimetry 2009, 34(1), 51-56; DOI: 10.1016/j.meddos.2007.12.003.

3. International Atomic Energy Agency (IAEA). Dosimetry of small fields used in external beam radiotherapy. Technical Reports Series 4832017. 
4. Griessbach, I.; Lapp, M.; Bohsung, J.; Gademann, G.; Harder, D. Dosimetric characteristics of a new unshielded silicon diode and its application in clinical photon and electron beams. Medical Physics 2005, 32(12), 3750-3754; DOI: 10.1118/1.2124547.

5. Di Venanzio, C.; Marinelli, M.; Milani, E.; Prestopino, G.; Verona, C.; Verona-Rinati, G.; Falco, M.D.; Bagalà, P.; Santoni, R.; Pimpinella, M. Characterization of a synthetic single crystal diamond Schottky diode for radiotherapy electron beam dosimetry. Medical Physics 2013, 40(2), 021712; DOI: 10.1118/1.4774360.

6. Bagalà, P.; Di Venanzio, C.; Falco, M.D.; Guerra, A.S.; Marinelli, M.; Milani, E.; Pimpinella, M-; Pompili, F.; Prestopino, G.; Santoni, R.; Tonnetti, A.; Verona, C.; Verona-Rinati, G. Radiotherapy electron beams collimated by small tubular applicators: characterization by silicon and diamond diodes. Physics in Medicine and Biology 2013, 58(22), 8121-8133; DOI: 10.1088/0031-9155/58/22/8121.

7. Di Venanzio, C.; Marinelli, M.; Tonnetti, A.; Verona-Rinati, G.; Bagalà, P.; Falco, M.D.; Guerra, A.S.; Pimpinella, M. Comparison between small radiation therapy electron beams collimated by Cerrobend and tubular applicators. Journal of Applied Clinical Medical Physics 2015, 16(1), 5186; DOI: 10.1120/jacmp.v16i1.5186.

8. Akino, Y.; Fujiwara, M.; Okamura, K.; Shiomi, H.; Mizuno, H.; Isohashi, F.; Suzuki, O.; Seo, Y.; Tamari, K.; Ogawa, K. Characterization of a microSilicon diode detector for small-field photon beam dosimetry. Journal of Radiation Research 2020, 61(3), 410-418; DOI: 10.1093/jrr/rraa010.

9. Weber C.; Kranzer, R.; Weidner, J.; Kröninger, K.; Poppe, B.; Looe, H.K.; Poppinga, D. Small field output correction factors of the microSilicon detector and a deeper understanding of their origin by quantifying perturbation factors. Medical Physics 2020, 47(7), 3165-3173; DOI: 10.1002/mp.14149.

10. Francescon, P.; Kilby, W.; Noll, J.M.; Satariano, N.; Orlandi, C. Small field dosimetry correction factors for circular and MLC shaped fields with the CyberKnife M6 System: evaluation of the PTW 60023 microSilicon detector. Physics in Medicine and Biology 2020, 65(1), 01NT01; DOI: 10.1088/1361-6560/ab6154.

11. Poppinga, D.; Kranzer, R.; Ulrichs, A.B.; Delfs, B.; Giesen, U.; Langner, F.; Poppe, B.; Looe, H.K. Three-dimensional characterization of the active volumes of PTW microDiamond, microSilicon, and Diode E dosimetry detectors using a proton microbeam. Medical Physics 2019, 46(9), 4241-4245; DOI: 10.1002/mp.13705.

12. Akino, Y.; Das, I.J.; Fujiwara, M.; Kaneko, A.; Masutani, T.; Mizuno, H.; Isohashi, F.; Suzuki, O.; Seo, Y.; Tamari, K.; Ogawa, K. Characteristics of microSilicon diode detector for electron beam dosimetry. Journal of Radiation Research 2021, 62(6), 1130-1138; DOI: 10.1093/jrr/rrab085.

13. Khan, F.M.; Doppke, K.P.; Hogstrom, K.R.; Kutcher, G.J.; Nath, R.; Prasad, S.C.; Purdy, J-A.; Rozenfeld, M.; Werner, B.L. Clinical electronbeam dosimetry: Report of AAPM Radiation Therapy Committee Task Group No. 25. Medical Physics 1991, 18(1), 73-109; DOI: 10.1118/1.596695.

14. International Atomic Energy Agency (IAEA). Absorbed dose determination in external beam radiotherapy. Technical Reports Series 3982000.

15. Schönfeld, A.; Poppinga, D.; Kranzer, R.; De Wilde, R.L.; Willborn, K.; Poppe, B.; Looe, H.K. Technical Note: Characterization of the new microSilicon diode detector. Medical Physics 2019, 46(9), 4257-4262; DOI: 10.1002/mp.13710.

16. Castro, P.; García-Vicente, F.; Mínguez, C.; Floriano, A.; Sevillano, D.; Pérez, L.; Torres, J.J. Study of the uncertainty in the determination of the absorbed dose to water during external beam radiotherapy calibration. Journal of Applied Clinical Medical Physics 2008, 9(1), 70-86; DOI: 10.1120/jacmp.v9i1.2676. 
17. Bouchard, H.; Seuntjens, J.; Kawrakow, I. A Monte Carlo method to evaluate the impact of positioning errors on detector response and quality correction factors in nonstandard beams. Physics in Medicine and Biology 2011, 56 (8), 2617-2634; DOI:10.1088/0031-9155/56/8/018. 